\title{
دور مؤسسات التعليم العالي في تنمية المجتمع في ضوء تطبيق معايير الجودة
}

\author{
د. آمال محمد عامر 1 \\ كلية الآداب- جامعة مصراتة
}

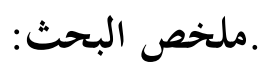

تتناول هذه المقاربة هذه البحثية إلى إبراز دورمؤسسة التعليم العالي في تنمية المجتمع في ضؤ تطبيق إدارة الجودة.وتوسيع آفاق المجتمع المعرفية و الثقافية ؛ من خلال جهود مؤسساته في تخريج كوادر بشرية تمتلك المعرفة و العلم والكفاءة ، قادرة على العمل في مختلف التخصصات العلمية ؛ حيث توظف طاقاتا و إمكاناتا لتحقيق تنمية المجتمع المستدامة باعتبارها هذه الكوادر"رأس المال البشري"في عملية التنمية وخدمة المجتمع؛ وهذه رسالة الجامعة رسالتها التي تعتبرأداة لصنع قيادات المجتمع الفنية والفكرية والمهنية.في الوقت الذي نشهد فيه تنامي ظهوروتأثير بجتمع المعلومات والمعرفة بكل تأثيراته على التغيرات الاجتماعية.ومن هنا يأتي الدورالفاعل لمؤسسات التعليم العالي في تزويد المجتمع بمخرجات تتلاءم مع طبيعة العصرلتحقيق تنميته الشاملة الإجتماعية و الاقتصادية و المعرفية و السياسية. الكلمات المفتاحية: تنمية المجتمع، رأس المال البشري، الجودة

a.benamer@art.misuratau.edu.ly ${ }^{1}$ 


\section{Abestract}

The aim of this research approach is to highlight the role of the Higher Education Institution in the development of society in the application of TQM and to expand the horizons of the knowledge and cultural society through the efforts of its institutions in the graduation of human cadres possessing knowledge, science and competence, It employs its energies and potentials to achieve the development of sustainable society as these cadres "human capital" in the process of development and community service; this is the message of the university its mission, which is considered a tool for the manufacture of artistic, intellectual and professional community leaders. Matt knowledge with all its effects on Alajtmaih.omn changes here Alldoralvaal comes to higher education institutions in providing community outcomes consistent with the nature of Alasrlthakiq comprehensive social, economic, political and cognitive development.

\section{1}

$$
\begin{aligned}
& \text { يعدبر التعليدم الجامعي من أهم مراحل التعليم التي تعمل على إعداد الكوادر الدؤهلة } \\
& \text { لإحداث التغييرالإيجابي في المجتمع وركيزة أساسية لتحقيق تزمية شاملة،وهواستردمار في } \\
& \text { العزصر البشري الذي يعتبر أهم موارد المجتمع يملكها أي مجزمع يسعى للوصول إلى تحقيق } \\
& \text { التنمية الشاملة والمستدامة.و هذا ما دفع بالدجزمعات الدتقدمة إلى جعل التعليم الجامعي } \\
& \text { من أهم أولوياتها ، و بقدر تطور التعليم العالي وارتقائه وتنوع مخرجاته وضمان مخرجات } \\
& \text { مؤهلة قادرة عل قيادة التزدية الشاملة،يتحقق الاستغلال الأمثل لموارد المجتمع البشرية التي } \\
& \text { تقود مسؤولية العملية التنموية.ومن هنا تعتبرالجامعة وسيلة تطويرالمهزمع. }
\end{aligned}
$$


على الرغم من التطورالملحوظ الذي شهله التعليم العالي إلا أن مساهته في تنمية المجتمع لازالت ليست ذات فاعلية وذلك بسبب ضعف الربط بين مؤسسات التعليم العالي ومؤسسات الجمتمع محا أدى إلى عدم الوقوف على متطلبات واحتياجات هذه المؤسسات

$$
\text { وعدم تواؤمها مع خخرجات التعليم العالي. }
$$

أهمية البحث: -تطويرؤسسات التعليم العالي بما يؤدي إلى الاسهام في تنمية المجتمع -تطبيق معايير إدارة الجودة في مؤسسات التعليم العالي بما يؤدي الى ان تلبي مخرجات التعليم العالي متطلبات المتمع

-تقوية العلاقة بين مؤسسات التعليم العالي والمجتمع بما يؤدي الم تنمية الموارد البشرية في ضؤ تطبيق معايير إدارة للجودة. : 1 2 : 2 أسئلة البحث

كيف يمكن لمؤسسات التعليم العالي المساهمة في تنمية المجتمع باستخدام أسلوب إدارة الجودة . 1 - n

كيف يمكن تطويرالتعليم العالي من اجل تحقيق تنمية المجتمع في ضؤ تطبيق معايير إدارة

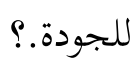
وما هي المتطلبات اللازمة لمؤسسات التعليم العالي للقيام بدورها في تنمية وتطوير المجتمع بكفاءة و فاعلية؟ 


\section{تهدف هذه الورقة البحثية إلى:}

- تسليط الضوء على مفهوم المسؤولية المجتمعية لمؤسسات التعليم العالي ودورها في التنمية الشاملة المستدامة في ضوء تطبيق معايير الجودة.

- إبراز أهمية قيام مؤسسات التعليم العالي بدورها في تنمية الجمتمع عن طريق الاستثماريي رأس المال البشري في ضوء تطبيق معايير الجودة.

-التعرف على فلسفة و تطبيق معايير الجودة ومعوقاتما في التعليم العالي. -التعرف على متطلبات تطبيق معايير إدارة الجودة في التعليم العالي.

-التعرف على المتطلبات اللازمة للتعامل مع معوقات أداء الجامعة لدورها في التنمية | ملمتمعية.

1. 4 مصطلحات البحث وحدوده:المسؤولية المجتمعية،الاستثماريف رأس المال البشري،

فلسفة الجودة،فلسفة التعليم العالي.2

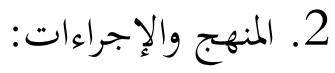

اعتمدت الباحثة المنهج الوصفي التحليلي النظري الذي يعتمد على تحليل مفهوم الجودة ومتطلبات استخدامها في التعليم العالي لتحسين المخرجات التعليمية وتعزيز العلاقة مع مؤسسات المجتمع لتحقيق تنمية المجتمع. 
يعد مفهوم إدارة الجودة الشاملة "أحد المفاهيم الإدارية الحديثة،حيث تمزج مابين الوسائل الإدارية والاعمال الابتكارية والمهارات الفنية من اجل رفع مستوى الإدارة."

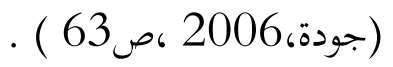

ومفهوم إدارة الجودة الشاملة هو ترجمة للتعبير:Total Quality Management وهناك من تناول التعريف بحسب كل عنصر. وعرف الإدارة بأها: "التطويرللمؤسسة من أجل رفع متواصل لمستوى الجودة" (جودة،2006،ص64 )؛وعرَّف الجودة Quality بأها :"متطلبات واحتياجات المستفيد" (جودة،2006،ص64 )؛وعرف الشاملة Total بأها: "السعي من أجل تحقيق الجودة في كل مظاهر العمل وجوانبه،ابتداء من متطلبات المستفيد وانتهاء بالتقويم لمدى رضى المستفيد عن ما تقدمه له المؤسسة من خدمات."

$$
\text { (جودة، (ج2006 ،ص265 ). }
$$

ماهية وفلسفة وأهداف إدارة الجودة الثاملة بالتعليم العالي: ماهية الجودة الشاملة بالتعليم العالي:

إن رسالة ومسؤولية مؤسسة التعليم العالي لم تعد تقتصرعلى احتياجات الطلاب، بل تمدف الى ضمان التحسين المستمر لأداء وقدرات الطلاب البحثية ،من خلال تحقيق فلسفة إدارة الجودة الشاملة في المؤسسة.ومن هناك ارتبط مفهوم الجودة الشاملة للتعليم العالي بكل عناصرالعملية التعليمية في المؤسسة وليس فقط بالطلاب. 
عدد خاص بالورقات العلمية المقدمة للمؤتمر الدولي الثاني للتعليم في ليبيا، مصراتة، ليبيا، مارس 2019

ويعرف البعض الجودة الشاملة للتعليم العالي بأها: "ترجمة احتياجات وتوقعات خريجي المؤسسة كمخرجات لنظام التعليم في كل كلية الم خصائص ومعايير محددة في الخريج تكون أساسا لتصميم وتنفيذ برامج التعليم مع التطويرالمستمرها" (بدوي،2010 ،ص56 ) . وتعرف أيضا على أها:" استثمار مهم لأن ما تقدمه الجامعة من اجل تلبية رغبات وحاجات زبائنها من الطلاب والمؤسسات يجعلها في تغيرمستمر مع رغبات المؤسسات هذا الامر يساعد على ضمان الخدمة المقدمة." (بدوي،2010 ،ص66 ). ومن هنا كانت للجودة الشاملة في التعليم العالي معايير علمية من اجل تحقيق استثمار في الموارد البشرية باعدادها وتطويرقدراتها لتلبية متطلبات المجتمع واحتياجات مؤسساته لتحقيق التنمية المجتمعية الشاملة.

\section{الفلسفة التي تتأسس عليها الجودة الشاملة في التعليم العالي:}

تتأسس إدارة الجودة الشاملة على فلسفة التطويروالتحسين المستمروالابداع والابتكاروخلق روح الابداع في العمل والعاملين.فالشخصية المبدعة الخلاقة تضيف للعمل وتبدع في تطويره بدلا من ان تكون شخصية تنفذ وتؤدي المهام دون تفكيرفي الإضافة والابتكار.وهي فلسفة تلتقي مع الدورالحيوي لمؤسسة التعليم العالي في إعداد الكوادروالخبرات بما يمقق تنمية

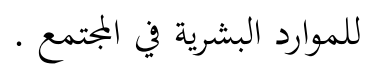

والطالب الباحث يتم اعداده في مؤسسة التعليم العالي باعتبارها مصنعا للعقول المفكرة المبدعة التي ستعمل على تطويرالمؤسسات بالمجتمع بما يؤدي الى تحقيق تنمية بجتمعية حقيقية.وباعتبارالطالب الباحث ثروة بشرية تعد ركيزة أساسية في تنمية المجتمع. 
ومن هنا اعتمدت الإدارة الشاملة للجودة على تطويرالمهارات ليس فقط من اجل تحسين الأداء ورفع مستوى الإنتاجية والمخرجات ،وانما من اجل تنمية شخصية تكون فاعلة تؤمن بقدراها ،وتعمل على تطويرها وتنميتها وبالتالي تتعزز لديها القدرة على الابتكار والتجويد في العمل والأداء.ومن هنا تصبح مسؤولية الجودة مناطة بجميع العاملين في المؤسسة مشاركة وفاعلية.

\section{أهداف الجودة الثاملة في التعليم العالي:}

"-دراسة متطلبات المجتمع واحتياجات مؤسساته ووضع الاستراتيجيات والدراسات من أجل تلبيتها. - تنمية القيم التي تتعلق بالعمل الجماعي والعمل بروح الفريق. - تحقيق الإحساس بالمشاركة في تطويرالمؤسسة لدى جميع العاملين بالمؤسسة. - تعزيزالاهتمام عند الباحثين على ربط البحث النظري بواقع المجتمع ومتطلباته ومشكلاته. - تققيق جودة الباحث في الجوانب البحثية والمعرفية والمهارات وتعزيزالقيم الأخلاقية لديه. - تطويرالمهارات لدى جميع افراد المؤسسة التعليمية. -العمل على التقييم والتقويم المستمر لعملية التعليم والبحث ومدى النجاح في ربطها بمشكلات ومتطلبات المجتمع. - تعزيزارتباط مؤسسة التعليم العالي بالمجتمع ومؤسساته ومتطلباهما لتكون المخرجات ملبية

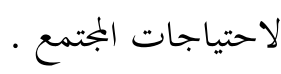


-ضمان استمرارية الميزة التنافسية مع مؤسسات التعليم العالي ذات الجودة العالية في الدول

$$
\text { الأخرى." (أبوالنصر،2008 ،ص } 43 \text { ). }
$$

\section{فلسفة وأهداف مؤسسات التعليم العالي وعلاقتها بالجمتمع:}

تعمل مؤسسات التعليم العالي على توفير تعليم متقدم لأشخاص لديهم القدرات العلمية

والفكرية لمتابعة دراسات متخصصة. ونشرالمعرفة في مجالاتها الذظرية والتطبيقية، وتهيئة الظروف الهناسبة لتزمية الخبرات الوطنية .

ويحتوي التعليم العالي على مجموعة من الدؤسسات الجامعة، المراكز البحثية، المعاهد العليا، مؤسسات البحث العلمي؛ولهذا فإن التعليم العالي بهذا المعنى هو مفهوم أوسع، يشدل العديد من المؤ سسات التعليمية والدحثية.وعلى الرغم من تعدد الزوايا التي يدكن من خلالها الذظر إلى مؤسسات التعليم العالي "ومن زاوية تعليمية هي مؤسسة تعليمية تقوم بالبحث العلمي، ومن زاوية اجزماعية هي مؤسسة اجزماعية توجه أبحاثها لتلبية متطلبات

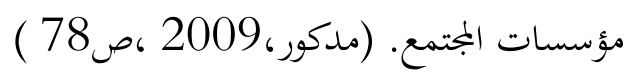

إلا أن جميع برامج التعليم العالي على تعددها تلتقي في تحقيق خدمة المجتمع والعمل على رفد مؤسساته بالكوادرالمؤهلة.ذلك ان الفلسفة التي تقوم عليها مؤسسات التعليم العالي هي الاستثمارفي العنصرالبشري أي رأس المال البشري للمجتمع.فهي تعتبرالانسان هدف الحياة في المجتمعات،فكل شيء من اجل خدمة الانسان باعتباره القيمة العليا في الحياة. وانطلاقا من هذه الفلسفة تضع مؤسسات التعليم العالي أهدافها وترسم استراتيجيتها التي تطويرالكفاءات وتحمل المسؤولية المجتمعية.ويعرف عبدالرحمن بدوي المسؤولية المجتمعية بأها "إقرار الفرد بما يصدر عنه من أفعال واستعداده لتحمل نتائجها". (بدوي، 1981 
،ص134 ).لذلك تستجيب مؤسسات التعليم العالي لمتطلبات المجتمع،وتتفاعل مع البيئة من اجل تحقيق اهداف مشتركة،وتضع الاستراتيجيات من اجل احداث التغييرالايجابي بالمجتمع وتحقيق التنمية المجتمعية.

\section{أهداف مؤسسات التعليم العالي:} توفير أعضاء الهيئة التدريسية والباحثين الذين يتطلبهم قطاع التعليم العالي. - تطوير شخصية الباحث، وتعزيز قدراته على التحليل والنقد والمبادرة والإبداع والحوار الإيجابي.

- تحديد موضوع الرسائل العلمية والأطروحات الخاصة بالدراسات العليا، بما يضدن تزمية البحث المرتبط بمدطلبات خطط التزمية المجتمعية.

- إكساب الطلاب الباحثين طرق التفكير العلمي يجعله يسعى للتطور والتجديد ويشارك في إحداث التقدم للدجزمع،

- إمـاد سوق العمل بذوي الاختصاصـات الهامة الذين تحتاجهم قطاعات الدجزمع متابعة الخريجين في مواقع عملهم.

- تعزيز التعاون بين مؤسسات التعليم العالي ومؤسسات المجتمع، والعمل على خلق الإتصال لتحقيق التعاون المثمر وتكامل الخبرات بما يؤدي إلى خدمة كلا من الجامعة

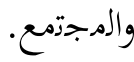

ولذلك تعتبرمؤسسة التعليم العالي مركزا علميا ومؤسسة لإزتاج المعرفة وتطويرها وتوظيفها في خدمة الدجتمع،وإعداد الباحثين وتقديم تعليم وتدريب يهدف إلى تعزيز 
القدرة على التفكير والدناقشة والتحليل،وتنمية المهارات من اجل الاستعداد للعمل في الدؤسسات، وقد عملت العديد من الدجزمعات على تنمية المؤسسات البحثية وتمويلها،بما أها تشكل الدعامة لتطويرالمجتمع. "فالتطورالمعرفي الكبيروالتقدم التقني الذي يشهده هذا العصر،جعل من أولوية مسؤوليات مؤسسة التعليم العالي النهوض بمجتمعها ومتابعة التطورالتكنولوجي والمساهمة فيه و تطوير البرامج البحثية، و إعداد الكوادر البشرية علميا و فنيا و إداريا واجتماعيا ، في ضوء التطور العلمي والتكنولوجي و متطلباته."

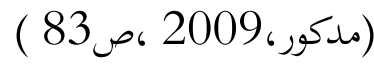

\section{دور مؤسسات التعليم العالي في تنمية الجمتمع:}

يعتبر التعلديم العـالي أحسد أهـم الفاعلين في تحقيق التزديـة الشاملة في المجتمع، حيـث يعمل على إعـداد وتطوير القـدرات والمهـارات البشـرية، وبواسطة التعلديم العـالي يستطيع البجتمع أن يواكـب حركـة التقـدم العلمي والتكذولوجي التي يشهدها العالم "وتقدم الدول لم يعد يقتصر على مـا تمتلكه من موارد طبيعية ورأس مـال مـادي، بل أصبح بنـاء الأمم الحديثة مـتبط بمـدى توفرهاعلى الكفــاءات المؤهلة ذات التأهيل العـالي، والتي بإمكانها قيادته عـن طريق رسم السياسـات والاستراتيجيات والعمـل على تنفيذها." (بجاهد،2011 ،ص47 ).وهنا يبرز الدور الجوهري الذي تقوم به مؤسسات التعليم العالي في تزدية قدرات الأفراد ،وتزويدهم بالمعارف وتطوير قدراتم التي تمكنهم مس الخلق والإبداع، واعتبارهم الثروة الدزتجة في كافة الدجالات والتي يراهن عليها المجتمع ويعتمد عليها من اجل تحقيق التنمية الشاملة والمستدامة. 
إن التعليم أصبح يُنظرإيه على انه استذمار لديه عائد مادي واجزماعي مهم، بفعل التطورات التي حققتها الدجزمعات الدتقدمة زتيجة النهوض بمدظومتها التعليمية. "فهو استذمار يتحمل جزء كبيرا من مسؤولية تحقيق التزمية الشاملة ،بمعناهـا الو اسـع الـذي يشـمل جميع نـواحي الحياة، بمـا فيها التزميسة الاقتصادية، الاجزماعية، الثقافية، والبشرية." (خليل،2011 ،ص95 ) وحتى يدم تحقيق الدزمية المجتمعية الشاملة، يتطلب ذلك الربط بين مؤسسات المجتمع وبين مؤسسات التعليم العالي حتى تنسجم استراتيجية مؤسسة التعليم العالي مع خطط التزمية الاقتصادية والاجزماءية .

\section{التعليم العالي في خدمة المجرتمع :}

تختص مؤسسات التعليم العالي بالدحث العلمي في سبيل خدمة الدجزمع والارتقاء به ،وتزمية القيم الإنسانية وتزويد البجتمع بالخبراء والدختصين في مختلف المجالات،وإعداد الإنسان المزود بالمعرفة وطرق البحث الحديثة ، ولتحقيق ذلك كان لزاما على الجامعة أن تقوم بإعداد الدوارد الإشرية ذات المهارة والكفاءة العالية للدجزمع ليقوموا بمسؤوليتهم بخدمة

$$
\text { الدجزمع وإحداث تغيير اجزماعي إيجابي. }
$$

و يمكن لمؤسسات التعليم العالي أن تساهم في خدمة الدجزمع وتزديته من خلال :- تقديم الخبرة إلى الدؤسسات في المجتمع. - القيام بالدحوث التطبيقية التي تعالج مشكلات الدجزمع وتساهم في حلها. - الدشار كة في الذدوات العلدية التي تذظم في مؤسسات غير جامعية. -عمل الدورات التدريبية لتكوين الكوادر العلمية التي تديرالمؤسسات. 
- تأليف الكتب والقيام بالترجمة ونقل المعارف من اللغات الأجذبية إلى اللغة العربية. -ربط المادة العلمية للمقرروالأبحاث بالفرد والمجتمع والبيئة بدد" لا من تدريسها بشكل يعزلها عن محيط الفرد في المجتمع. - ترسيخ قيم المواطنة الصادقة في الممارسات اليومية للأفراد والمؤسسات. - توفير الإمكانات البحثية و العملية لحل مشكلات المجتمع · -نشر المعرفة و تكوين العقلية الواعية لحل مشكلات المجتمع و البيئة المحيطة ، ومعرفة الأساليب الفنية المستخدمة ، وتتبعها لركب التقدم العلمي و التكنولوجي. -التنمية المهنية وتنمية بعض المهارات مثل: مهارات القيادة و الإتصال و غيرها. "إنّ خدمة المجتمع مسؤولية بجتمعية تقوم بها مؤسسات التعليم العالي؛فتعمل على تحديد الاحتياجات المجتمعية للأفراد و الجماعات و المؤسسات ، وتصميم البرامج التي تلبي هذه الاحتياجات عن طريق كلياتما و مراكزها البحثية المختلفة بهدف إحداث تغيرات تنموية وتحقيق التنمية المجتمعية الشاملة في المجالات المتعددة؛ وتمكين الأفراد والمؤسسات من تحقيق الاستفادة من الحخدمات المختلفة التي تقدمها الجامعة بوسائل متنوعة تتناسب مع ظروف وحاجات المستفيد." (مدكور،2009 ،ص68 )

وفي 26 يوليو سنة 2000 م انبثق في هيئة الأمم المتحدة الميثاق العالمي للمسؤولية المجتمعية "وهوعبارة عن مبادرة مواطنة طوعية متعلقة بالشركات ؛ يعرض تسهيلا و تعهدا من خلال عدة آليات،سياسة الحوار، المعرفة،و مشاريع شراكة، ويعتمد هذا الميثاق على المسؤولية و المشاركة في الأداء الجوهري المتعلق بالمسؤولية الاجتماعية العامة بما في ذلك 
شفافية الشركات والمؤسسات والقوى العاملة و المجتمع بمشاركة المبادئ المستند عليها

$$
\text { الميثاق." (مكتب العمل الدولي،2006 ،وثيقة رقم01 ) }
$$

إن مؤسسات التعليم العالي لاتنفصل عن المجتمع،وكل تطوير تقوم به الجمامعة باستخدام إدارة الجودة الشاملة في اساليبها وبرابجها ومدخلاتما ومخرجاتما ،ينعكس إيجابيا على المجتمع ويؤدي الم الدفع بالتنمية الشاملة.

من هنا يكون وضع خطة للتعليم العالي تستهدف تحقيق أهداف رئيسية منها : - التوسع في التعليم في ضوء حاجات المؤسسات ومتطلبات التزمية. - على مستوى عضو هيئة التدريس، يذبغي أن يواكب التغيرات العلمية الهذسارعة ويطور من أدائه. - على مسدوى الجامعة مراجعة وتطوير أزظمتها وقواذينها إداريا ومهزيا بما يحقق تطويرا في المرافق والخدمات، الرضا الوظيفي، ووسائل الاتصال - على هددتوى الطالب الجامعي، يذبغي أن تطويرقدرات ومهارات الطالب حتى يكون عنصرا فاعلا في تشكيل منهج ومحاور المادة العلمية، وإبداء وجهة الزظر، وبناء شخصية علمية مدتقلة قادرة على إبداء الرأي ومحاورة الآخرين. - إعداد العنصر البشري القادر على إحداث التنمية المنشودة من خلال إعداد الكوادر القادرة على مواجهة التغييرات العلمية و التكنولوجية المعاصرة. - تطوير وربط المناهج بأهداف التزمية . 
- القيام بالبحوث والدراسات التي تساءد على إيجاد الحلول لـختلف المشكلات في - المجتمع

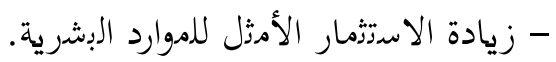
- تلبية متطلبات المجتمع من اجل تحقيق التنمية المستدامة متطلبات تطبيق إدارة الجودة الشاملة في مؤسسات التعليم العالي: لكي تتحقق الجودة الشاملة وتكون قابلة للتطبيق العملي في الدؤسسة الجامعية، فهي بحاجة إلى إحداث مرطلبات أساسية ومن هذه المتطلبات :- وضع الخطط اللازمة لتزمية ثقافة الجودة . - تو جيه الزشاطات الدبذولة لتطبيق إدارة الجودة الشاملة. - تفعيل جهود كافة أفراد الجامعة لتحقيق هدف إدارة الجودة الشاملة. - توفير الدوارد المادية والبشرية لتنفيذ فلسفة إدارة الجودة الشاملة - وضع الأهداف السنوية لإدارة الجودة الشاملة - تصديم البرامج التدريبية لقيادات الجودة وفرق العمل. - توثيق الصلة بين الجامعة والمؤسسات الأخرى - تحديد متطلبات المؤسسات بالمجتمع 
إن تربني أسلوب إدارة الجودة الشاملة في التعليم يعتبر أسلوبا فعالا لتحقيق التطويرالمستمر بمدسدوى التعليدم العالي، وتعزيز العلاقة بين التعليم العالي ومؤسسات المجتمع من اجل الوقوف على متطلباتما وتحقيق التنمية المجتمعية الشاملة. ومن أساليب إدارة الجودة الشاملة لضمان تحقيق أهدافها:-نظام الاقتراحات للعاملين والطلاب وهيئة التدريس ودراسة هذه الاقتراحات. - متابعة البيانات والاحصائية ومعالجة الإشكاليات. -حل المشكلات بتكوين لجنة لمناقشة المشكلات واقتراح الحلول من اجل تحسين الجودة. -عمل مقارنة والبحث عن افضل الأساليب التي تقوم بها مؤسسات منافسة من اجل تحقيق التطوروالتحسين وضمان الميزة التنافسية.

-العمل على مشاركة العاملين وكل عناصرالعملية التعليمية والمؤسسات المستفيدة من مخرجات التعليم بجيث تنطوي الجودة الشاملة على مسؤولية المشاركة الشاملة لضمان الاستمرارية في التطوير.

وبالتالي تكون الجودة الشاملة جزء رئيسي من استراتيجية مؤسسة التعليم العالي ومسؤولية يشترك فيها الجميع وتفاعل إيجابي بين المدخلات والمخرجات للمؤسسة ونظامها التعليمي. معوقات تطبيق الجودة الشاملة في مؤسسة التعليم العالي:رغم أهمية إدارة الجودة الشاملة في مؤسسة التعليم العالي إلا أن تطبيقها قد يواجه العديد من الصعوبات ومن أهم هذه الصعوبات:- 
- نقص البيانات من أجل الوصول إلى تحسين جوازب العمل والهناخ العلمي من حيث الأبحاث لتوجيه السياسات لربطها بالمجتمع. -عدم مشاركة جميع العاملين في تطبيق إدارة الجودة الشاملة. ومسدوى جودة الخدمة

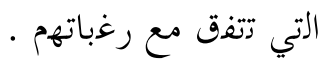

- غياب الربط بين الجامعة وقطاعات سوق العمل من حيث مدى تطور الدناهج طبقا

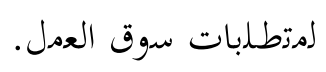
-عدم توفراميزانية الكافية. - ضعف الربط مع مؤسسات المجتمع لمعرفة متطلباتا واحتياجاتا. -نقص إنتاجية البحوث التي ترتبط بمتطلبات ومشكلات المؤسسات في المجتمع. -غياب الربط بين مؤسسة التعليم العالي ومؤسسات المجتمع عبر الوسائط الذكنولوجية الحديثة .مما أدى إلى غياب البيانات الشاملة عن مؤسسات المجتمع واحتياجاتما. -غياب التخطيط الاستراتيجي للمؤسسة من اجل ان تكون المساهمة في التنمية المجتمعية هدفا رئيسيا من اهداف المؤسسة. غياب المؤشرات عن مدى تحقيق مؤسسة التعليم العالي لخدمة الدجزمع مثل: -مدى استجابة البحوث لاحتياجات مؤسسات المجزمع الخدمية والإنتاجية . 
• عدد الاستشارات العلمية التي قدمتها مؤسسات التعليم الجامعي لمؤسسات المجتمع . عدد الابتكارات والاكذشافات الجديدة التي حققتها الجامعة و تخدم أهداف المجتمع

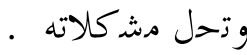

-الذسبة المدوية لأعضاء هيئة التدريس المشاركين في جمعيات خدمة المجتمع .

\section{5. الاستنتاجات والتوصيات:}

زخلص مدا سبق إلى أن عملية النهوض بالتزمية الشاملة للمجتمع لا تقتصرعلى الموارد المادية فقط، بل يعتبر العزصر البشري "رأس المال البشري" أساس التزمية ، ويقوم التعليم العالي بالدور الدحوري في تحقيق التنمية ،بما يعمل عليه من تكوين وصقل وتطويرمستمرلرأس المال البشري ،بتطبيق الجودة الشاملة التي تلتقي في فلسفتها وأهدافها مع الفلسفة والأهداف التي تتأسس عليها مؤسسات التعليم العالي . لذلك يتوجب على مؤسسات التعليم العالي انتهاج الاساليب الإدارية الحديثة، لمواكبة التطورات التكنولوجية والإدارية والعلمية المتزايدة، ويدثل تطبيق فلسفة إدارة الجودة الشاملة في مؤسسات التعليم العالي إحدى الأساليب الفعالة لتحقيق التطويرالذي يجعلها قادرة على تعزيز دورها في تحقيق التنمية الشاملة للمجتمع.

\section{توصيات البحث: ت ت}

-تطوير ثقافة الأفراد و المؤسسات حول التنمية المجتمعية وضرورة تحمل المسؤولية المجتمعية. -تضمين الموارد التعليمية خاصة المقررات الدراسية مفاهيما حول المسؤولية المجتمعية،والتنمية من خلال ربطها بمحتوى المقرر حيثما أمكن ذلك. 
-وضع الجامعات المسؤولية المجتمعية في صلب استراتيجياتا اعتمادا على دراسات و أبحاث للوقوف على احتياجات المجتمع وأهمية المشاركة في التنمية المجتمعية .

- إنشاء مركزمتخصص في الجامعة يعنى بالمسؤولية المجتمعية "إدارة خدمة المجتمع"وربطها بإدارة الجودة و متابعة أداء كل الوحدات في هذا المجال.

- إنشاء لجان استشارية مشتركة من الهيئة العاملة في الجامعة، وقيادات المجتمع مدن ذوي القرار لتحديد حاجات المجتمع و التعرف على مشكلاته. -توجيه الأبحاث الجامعية لحّل المشكلات المحلية سعيًا وراء خدمة المجتمع في حل المشكلات التي تعيق التنمية الشاملة. قائمة المراجع أولا: المراجع العربية: - أن -جودة،محفوظ،2006 ،إدارة الجودة الشاملة مفاهيم وتطبيقات،عمان،دار وائل للنشر. -بدوي،محمود، 2010 ،إدارة التعليم والجودة الشاملة،الإسكندرية،دارالتعليم الجامعي، - أبوالنصر،مدحت،2008 ،أساسيات إدارة الجودة الشاملة،القاهرة،دارجريرلنشر . - مدكور،علي،2009 ، الاستثمارفي التعليم بين مشكلات الحاضروتصورات المستقبل ، القاهرة،دارالفكرالعربي.

-بدوي،عبدالرحمن، 1981 ، الأخلاق النظرية،الكويت،دار سالم للطباعة والنشر . - مجاهد،محمد، 2011 استراتيجيات الجودة في التعليم،القاهرة،الدارالجامعية الجلديدة. 


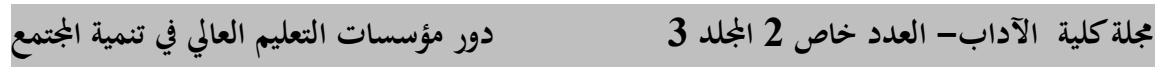
عدد خاص بالورقات العلمية المقدمة للمؤتمر الدولي الثاني للتعليم في ليبيا، مصراتة، ليبيا، مارس 2019 لئل 2019

- خليل،نبيل، 2011 ،إدارة الجودة الشاملة والاعتماد الأكاديمي في المؤسسات التربوية،القاهرة،دارالفجر للنشر.

-مكتب العمل الدولي، المبادرة المركزية بشأن المسؤولية الاجتماعية، الدورة 295. 